\title{
COMPLEX EFFECTS OF FREE STREAM TURBULENCE AND SURFACE ROUGHNESS ON THE TRANSITIONAL INTERMITTENCY
}

The comparison of transitional intermittency distributions in boundary layers on smooth and rough surface is presented. The conditional analysis of the instantaneous wall friction is applied on time records made in transitional boundary layers originating on smooth surface or surface covered by sand paper. Special procedures of measurement and evaluation are applied that allowed evaluations of turbulent and nonturbulent zone averages.

Keywords: By-pass transition, rough wall boundary layer, CTA anemometry, instantaneous wall friction, intermittency analysis

\section{Introduction}

The problem of joint action of wall roughness (WR) and free stream turbulence (FST) on the boundary layer development is very broad. The laminar turbulent transition plays a major role in boundary layer development so it deserves a special attention. Its relevance is in changes of skin friction, heat transfer and of tendency to flow separation that are caused by increased momentum and scalar diffusion after the transition. The mechanism of how an originally laminar boundary layer on a smooth surface is forced by disturbances penetrating into the layer from environment and of the corresponding routes to turbulent layer were described elsewhere, e.g. [1]. The opinion generally accepted is that the final phase of boundary layer laminar/turbulent transition starts with the occurrence of first turbulent spots regardless of the initial conditions.

The location of the laminar-turbulent transition region can be estimated from the distributions of characteristics derived from the mean flow measurements, especially if their course is known in laminar and turbulent states. This is the case of the flat plate boundary layer. The skin friction coefficient distribution seems most suitable for this purpose.

This paper is a follow up to the investigations of mean flow field in the zero pressure gradient boundary layers on plates with smooth surface and plates with surfaces covered by sandpapers (grits 60, 80 and 100) in turbulent free stream e.g. [2]. The skin friction coefficient value was derived from the mean velocity profile. Then the location of transitional region was carefully estimated by the diversion of the skin friction coefficient distribution from the Blasius solution [3] and by the attachment of the distribution to the Ludwieg and Tillmann empirical curve [4]. The accuracy of this procedure is generally weak, namely in rough wall boundary layers, because of the measurement scatter, of a sparse net of measurement, of a slow change of $C_{f}$ in the streamwise direction, etc. Fig. 1 illustrates this problem. Intensity Iu, length parameter Le and surface property are described successively in square brackets in caption Fig. 1. Hence it was decided to perform the transitional intermittency measurements in the investigated boundary layers.

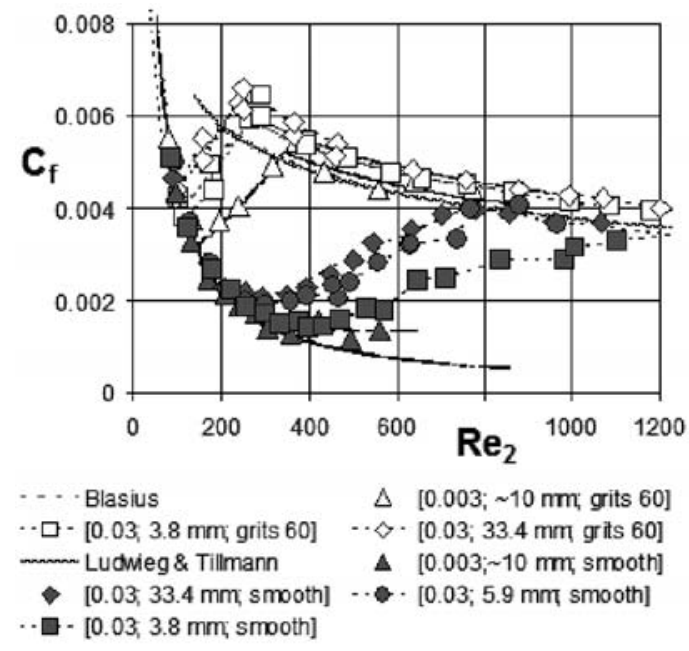

Fig. 1 Skin friction coefficient distributions on smooth and rough flat plates

\footnotetext{
* Ondrej Hladik, Pavel Jonas, Oton Mazur, Vaclav Uruba

Institute of Thermomechanics AS CR, v.v.i., Praha, Czech Republic, E-mail: hladik@it.cas.cz
} 


\section{Experimental facility and methods}

Investigations of boundary layers developing on smooth or rough plates were performed in the close circuit wind tunnel $(0.5$ $\times 0.9 \times 2.7 \mathrm{~m}^{3}$ ) of the IT AS CR, Prague, Fig. 2 .

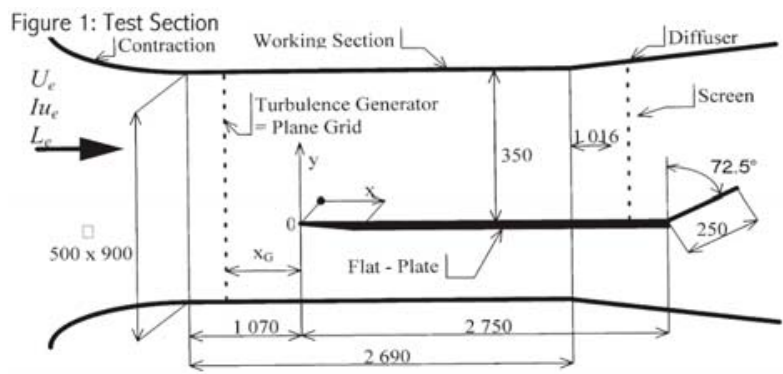

Fig. 2 Scheme of the working section of the wind-tunnel IT AS CR, Prague

The mean velocity of external stream was constant $( \pm 0.5 \%)$ about $5.0 \mathrm{~m} / \mathrm{s}$, during every measurement in the individual profiles of boundary layer in the given configuration.

Boundary layers were developing either on the original smooth plate with the special shape of the leading edge (L.E.), [5], or on the plate covered with sand paper grits 60 with the elliptic shape of the L.E., [2]. The maximum size of grains on sandpaper was chosen as the representative length of the sand paper roughness $s=(0.435 \pm 0.014) \mathrm{mm}$.

Free stream turbulence (FST) was either low - natural with the level $0.3 \%$ and the length scale of order $10^{-1} \mathrm{~m}$ or it was amplified by means of square mesh plain grids/screens. Three types of turbulence generators were used, each producing the intensity $I u=0.03$ and individually producing the dissipation length parameters $L e=$ $=3.8 \mathrm{~mm}, 5.9 \mathrm{~mm}$ or $33.4 \mathrm{~mm}$ at the plane of the L.E. $(x=0)$.

The mean flow boundary layer characteristics were evaluated from the mean velocity profiles measured in the investigated sections $x$ (const). Two single wire probes working in the CTA mode were used in the smooth wall configuration. The first reference probe, placed in a fixed position in the outer stream, serves as the indicator of the reference velocity. The second probe, the profile probe, was put into position by a traversing system in the streamwise direction $x$ and in the direction $y$ normal to the surface. The distance $y$ was measured with an accurate cathetometer. Digital records of the output signals were acquired simultaneously and then records of the relevant instantaneous velocities were evaluated using data from the calibration measurements performed prior to the experiment. Next, the correction [6] of the wall proximity effect on hotwire cooling was applied. The instantaneous wall friction time series $\tau_{w}(t)$ are the by-product of wall corrections.

Measurements in the rough wall configuration require more resistant probes than HW-probes for the essential measurements.
The couple of the flattened Pitot tube (probe tip: $0.18 \mathrm{~mm} \times 2.95$ $\mathrm{mm}$ ) and the round nosed static pressure probe (diameter $=1.8$ $\mathrm{mm}$ ) serves as the profile probe and the Pitot-static probe (diameter $=6 \mathrm{~mm}$ ) is the indicator of the reference velocity. The instantaneous wall friction measurement is carried out by means of a wall hot wire probe step-wise moving in the streamwise direction in the distance from the rough surface $y_{0} \sim 0.3 \mathrm{~mm}$. The probe (calibrated in advance) is attached to the three wheels truck connected to the traversing system. The probe is working in the CTA mode and the output voltage is recorded. Next, these records are digitally transformed using calibration parameters into the records of the local nominal instantaneous velocity. The CTA output signals processing follows assuming the adjusted y0 like that in the smooth wall case with one important difference. Irregular variations of the true distance $y_{w}$ from the adjusted one $y_{0}$ are of the order hundreds millimetre and vary with the location $x$. Thus an auxiliary local correction of $y_{w}$ must be made. The correction equals the time averaged skin friction coefficient determined from the mean velocity profile with the mean value of skin friction coefficient calculated from the CTA measurement in the given location $x_{j}$. The instantaneous wall friction $\tau_{w}(t)$ is evaluated using the corrected distance yw in the vicinity of $x_{j}$.

The instantaneous wall friction records $(25 \mathrm{kHz}, 750000$ samples, 16 bit) were utilized in a statistical analysis of the wall friction in the rough wall boundary layer like in the smooth one.

The applied method of the transitional intermittency analysis is Turbulent Energy Recognition Algorithm-Method, e.g. [7] and the procedure of is very similar to that described in [8] and [9]. The method consists of several consecutive steps. At the first, the obtained records of the instantaneous values of wall friction fluctuations $\tau_{w}^{\prime}$ are filtered by Butterworth filter with low pass frequency $1 \mathrm{kHz}$ to eliminate noise from the signal. At the second step, the detector function $D(t)$ is derived as to emphasize the differences of the signal time behaviour during turbulent and non-turbulent periods. The detector function is computed after the formula:

$$
D(t)=\left|\tau_{w}^{\prime} \cdot \partial^{2} \tau_{w}^{\prime} / \partial t^{2}\right|
$$

then the detector function is smoothed to eliminate scales much smaller than those to be recognized, thus the criterion function $K(t)$ is created. The criterion function, the threshold $T h$ and the indicator function $I(t)$ are evaluated successively.

$$
I\left(t_{i}\right)=0 \text { if } K\left(t_{i}\right) \leq T h ; I\left(t_{i}\right)=1 \text { if } K\left(t_{i}\right)>T h
$$

The indicator function allows to assort the whole record in the time intervals with turbulent structure $(I=1)$ and those with laminar/non-turbulent structure $(I=0)$. Finally the transitional intermittency factor is calculated (details are presented in [10]).

$$
\gamma=\frac{1}{m} \sum_{i=1}^{i=m} I\left(t_{i}\right) ; \quad m=750000
$$

This procedure was verified [11] comparing the dimensionless turbulent spot production rates $n^{*} \sigma$ evaluated by using both the intermittency analysis and the wavelet one [9]. At first the authors 
applied the method within the investigation of the smooth flat plate boundary layer developing in the FST with turbulence intensity $I u=0.03$ and different values of the FST length parameter [11]

\section{Results}

\subsection{Transition region limits and turbulent spot production}

Distributions of the transitional intermittency factor $\gamma$ against the displacement thickness Reynolds number $R e_{1}$ are shown in Fig. 3. They are in the conformity with Fig. 1 and clearly illustrate the significant effect of FST, surface property and their joint action. Results related to smooth wall layers are marked with grey marks, results related to rough wall layers are plotted with empty marks. The shape of marks differentiates after the type of FST (intensity Iu and the length parameter $L e$ ) as demonstrated in the caption of Fig. 1.

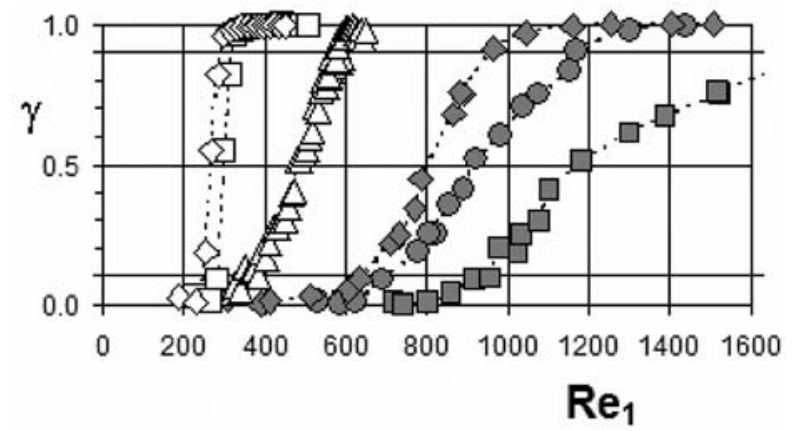

\footnotetext{
-ㅁ. $[0.03 ; 3.8 \mathrm{~mm}$ smooth $]-\cdot \circ \cdot[0.03 ; 5.9 \mathrm{~mm}$ smooth $]$

$\cdots \diamond \cdot[0.03 ; 33.4 \mathrm{~mm}$ smooth] - - $\square \cdot[0.03 ; 3.8 \mathrm{~mm}$ rough]

$\cdots \Delta \cdot[0.003 ; \sim 10 \mathrm{~mm}$; rough] $\cdots \diamond \cdot[0.03 ; 33.4 \mathrm{~mm}$; rough $]$
}

Fig. 3 Intermittency factor versus displacement thickness Reynolds number

The transitional intermittency factor gamma can be expressed after Narasimha [12] in the form involving the spot production rate $n$ (the number of spots occurring per unit time and space distance) and the Emmons [13] dimensionless propagation parameter $\sigma$ (including both the streamwise and lateral spot growth -, effect of drift)

$$
\gamma(x)=0, x \leq x_{t} ; \gamma(x)=1-\exp \left[-\left(x-x_{t}\right)^{2} n \sigma / U_{e}\right]
$$

Introducing the local Reynolds number into (4) we can define the dimensionless spot production parameter

$$
\begin{aligned}
& n^{*} \sigma\left(R e_{x}\right)=1-\exp \left[-\left(R e_{x}-R e_{t}\right)^{2} n^{*} \sigma\right], \\
& R e_{x}=x U_{e} / v, n^{*} \sigma=n v^{2} / U_{e}^{3}
\end{aligned}
$$

Thence the function $F(\gamma)$ suitable for the statistical estimates of the transition start and the value of parameter $n^{*} \sigma$ can be derived

$$
\begin{aligned}
& F(\gamma)=\sqrt{-\ln (1-\gamma)}=\sqrt{n^{*} \sigma}\left(R e_{x}-\operatorname{Re}_{x s}\right)= \\
& =a_{0}+a_{1} \operatorname{Re}_{x}
\end{aligned}
$$

The distributions of intermittency factor can be expressed in the universal form [12] by introducing the new variable $\zeta$

$$
\begin{aligned}
& \zeta=\left(\operatorname{Re}_{x}-\operatorname{Re}_{i r}\right) / \Delta \operatorname{Re}_{i r} ; \\
& \Delta \operatorname{Re}_{i r}=\operatorname{Re}_{x}(\gamma=0.9)-\operatorname{Re}_{x}(\gamma=0.1) ; \\
& \operatorname{Re}_{i r}=\operatorname{Re}_{x}(\gamma=0.5)
\end{aligned}
$$

Then the formula is valid at the configuration smooth surface and low FST level

$$
\gamma(\zeta)=1-\exp \left[-\alpha(\zeta+\beta)^{2}\right]
$$

The empirical parameters $\alpha$ and $\beta$ take the values $\alpha=1.42$ and $\beta=0.72$ after model proposed by Narasimha [12] or the values $\alpha=0.6$ and $\beta=1.05$ according to the model proposed by Johnson and Fashifar [14]. Formerly [11], the agreement of results obtained in smooth wall boundary layer at increased FST level with (8) was confirmed. The data received in rough wall layer under different FST, plotted in Fig. 4 demonstrate also their compatibility with the universal form (8).

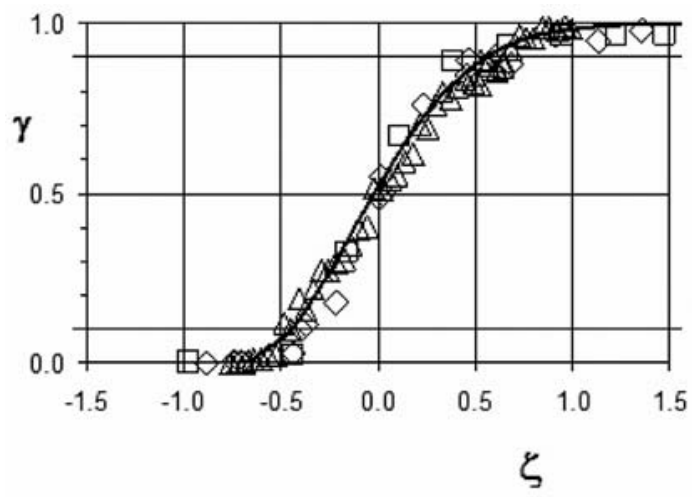

Fig. 4 Universal intermittency function

Starting from the equation (6) the relation can be derived between the dimensionless spot production rate and the Reynolds number defined with the length of transition region. Let the start of transition relates to $\gamma=\gamma_{s}$ and $R e_{x}=R e_{s}$ and similarly the end relates to $\gamma=\gamma_{c}$ and $R e_{x}=R e_{c}$. Substituting into (6) after some formal adaptations the relation is derived

$$
\begin{aligned}
n * \sigma & =\left(\sqrt{-\ln \gamma_{c}}-\sqrt{-\ln \gamma_{S}}\right)^{2} / \Delta R e_{t r}^{2} ; \\
\Delta R e_{i r} & =\left(R e_{S}-R e_{C}\right)
\end{aligned}
$$


the insertion of values $\gamma_{s}=0.1$ and $\gamma_{c}=0.9$ into (9) results in the formula

$$
n^{*} \sigma=1.42 / \Delta R e_{i r}^{2}
$$

The formula (10) and interpolation of Fransson et al [15] are plotted in Fig. 5 together with the authors' results. The received results agree well with the relation (10) regardless the boundary layer is developing on the smooth surface or on a rough one. Apparently turbulent spot production grows and the width of transition region shortens with increasing roughness and with growing FST.

\subsection{Conditional analysis of wall friction during laminar-turbulent transition}

Having determined the time behaviour of the indicator function $I(t)$, the record of the instantaneous wall friction $\left(\tau_{w}\left(t_{i}\right)\right)$ is possible to resolve in individual non-turbulent/laminar events and turbulent events. Every uninterrupted segment of record with $I\left(t_{i}\right)$ $=$ constant $(0$ or 1$)$ is named event.

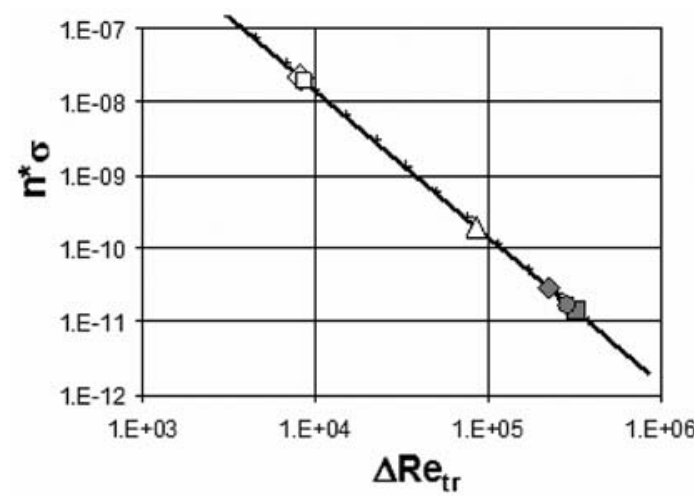

Fig. 5 Dimensionless spot production rate as function of transition length Reynolds number (symbols as in Fig. 1; Eq. (11) - straight line; interpolation of results [15] - crosses)

Thus it is possible to divide the total record $\left(\tau_{w}\left(t_{i}\right)\right)$ between the non-turbulent/laminar part (subscript L) and the turbulent one (subscript $\mathrm{T}$ )

$$
\begin{aligned}
& \left(\tau_{w}\left(t_{i}\right)\right), i=1,2, \ldots n=750000 \\
& \left(\tau_{w}\left(t_{i}\right)\right)_{L}=\left(1-I\left(t_{i}\right)\right) \tau_{w}\left(t_{i}\right) ; \quad\left(\tau_{w}\left(t_{i}\right)\right)_{L}=I\left(t_{i}\right) \tau_{w}\left(t_{i}\right)
\end{aligned}
$$

Apparently, any kind of the statistical analysis of every record could be done as customary at every experimental set up and in each cross section $x$. The analyzed wall friction records ( 750 thousands samples) were made at the same boundary conditions as the measurements plotted in Fig. 1. Here the attention will be concerned with the effect of boundary conditions on the mean time of the laminar $(\Delta t)_{L}$ and turbulent $(\Delta t)_{T}$ events duration and on the distributions of the wall friction conditional means $\left(\tau_{w}\right)_{L}$ and $\left(\tau_{w}\right)_{T}$.
The mean durations of the wall friction stay in non-turbulent condition $(\Delta t)_{L}$ and in turbulent condition $(\Delta t)_{T}$ are plotted in Fig. 6; right the results referring to the rough wall boundary layers and left the results referring to the smooth wall boundary layers. Laminar periods $(\Delta t)_{L}$ are of about two orders longer than the turbulent periods $(\Delta t)_{T}$ at the start of transition $x_{s}$. The opposite relations are at the transition completing. The courses are similar in smooth wall layers as in rough wall layers. Locations of maximum $(\Delta t)_{L}$ and minimum $(\Delta t)_{T}$ relate to the location of $\gamma=0$. The course of growing and decreasing of the discussed durations proceed in the streamwise similarly. An apparent difference between the smooth wall layers and the rough ones is ascertained in the events durations that are roughly of order longer in the smooth wall configuration. This can be attributed to the effect of numerous flow disturbances generated by wakes of individual sand grains on rough surface however a definite physical explanation of this phenomenon is not available yet. The distributions of the conditional mean wall friction in the investigated boundary layers versus the displacement thickness Re-number are shown in Fig. 7 in the same way as in the preceding Fig. 6. Presented are the results of measurements in boundary layers under boundary conditions that differ only in sur-face property - smooth or rough. Distributions after Blasius solution (full line) and after Ludwieg and Tillmann formulae (dashed line; necessary substitute actual values of the shape factor and the momentum thickness Re-number) are also plotted in Fig. 7.

The non-turbulent/laminar means $\left(\Delta \tau_{w}\right)_{L}$ follow the Blasius distribution up to the location where the intermittency factor becomes zero value. Afterwards the values $\left(\Delta \tau_{w}\right)_{L}$ continue at about constant level in smooth wall layers or start to grow in rough wall layers. The increase continues little beyond the end of transition region $\gamma=1$ and then the increase is possibly succeeded by the decrease (unfortunately measurements were closed too early already just after reaching locations $\gamma \sim 1$ ). The mean wall friction $\left(\Delta \tau_{w}\right)_{T}$ during turbulent events diverges from the Blasius distribution sooner than $\left(\Delta \tau_{w}\right)_{L}$. It diverges about in the section where the displacement Re-number reaches value 200. Afterwards the values $\left(\Delta \tau_{w}\right)_{T}$ continue at about constant level in smooth wall layers or start to grow in rough wall layers up to the end of transition region. This development is finished at reaching the level relevant to the distribution after Ludwieg and Tillmann empirical formula [4] evaluated with the valid values of the shape factor and momentum thickness Re-number.

\section{Conclusion}

The spot occurrence is more numerous in rough wall transitional boundary layer (RWL) than in the smooth wall layer (SWL) at otherwise equal boundary conditions. Owing to this transition starts sooner and the area of transition region is shorter in RWL than in SWL.

The increase of the free stream turbulence (FST) level Iu, in the plane of boundary layer origin $x=0$, amplifies the above-mentioned phenomena. The effect of FST length parameter Le on by- 

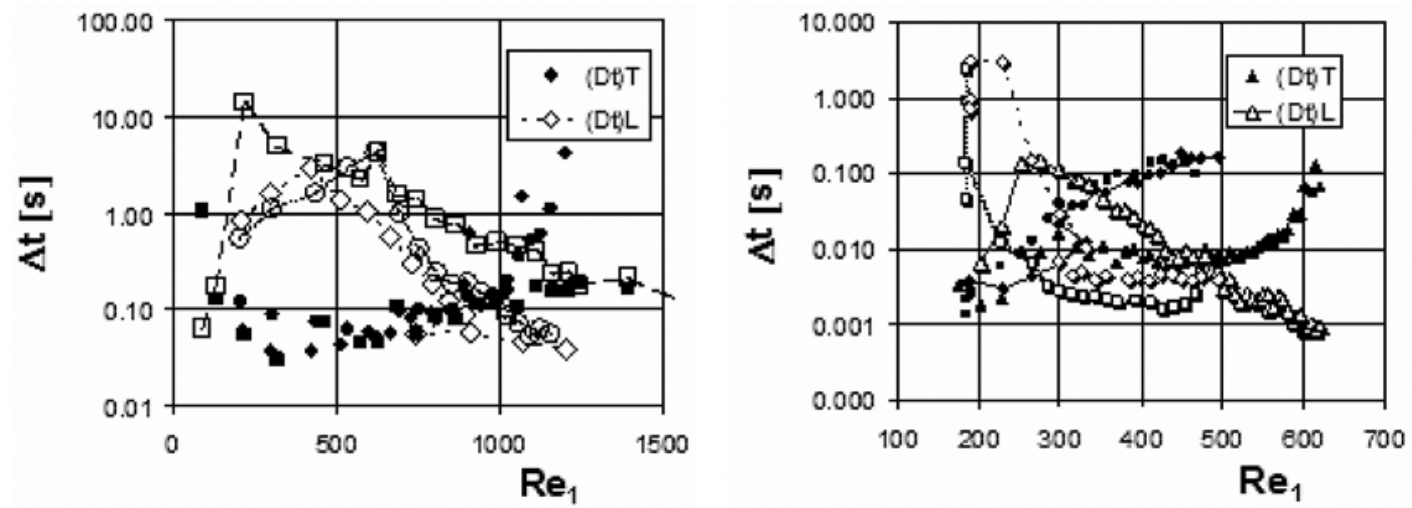

Fig. 6 The mean durations of the wall friction stay in non-turbulent condition $(\Delta t)_{L}$ and in turbulent condition $(\Delta t)_{T}($ left smooth surface; right rough surface; symbols as in Fig.1)
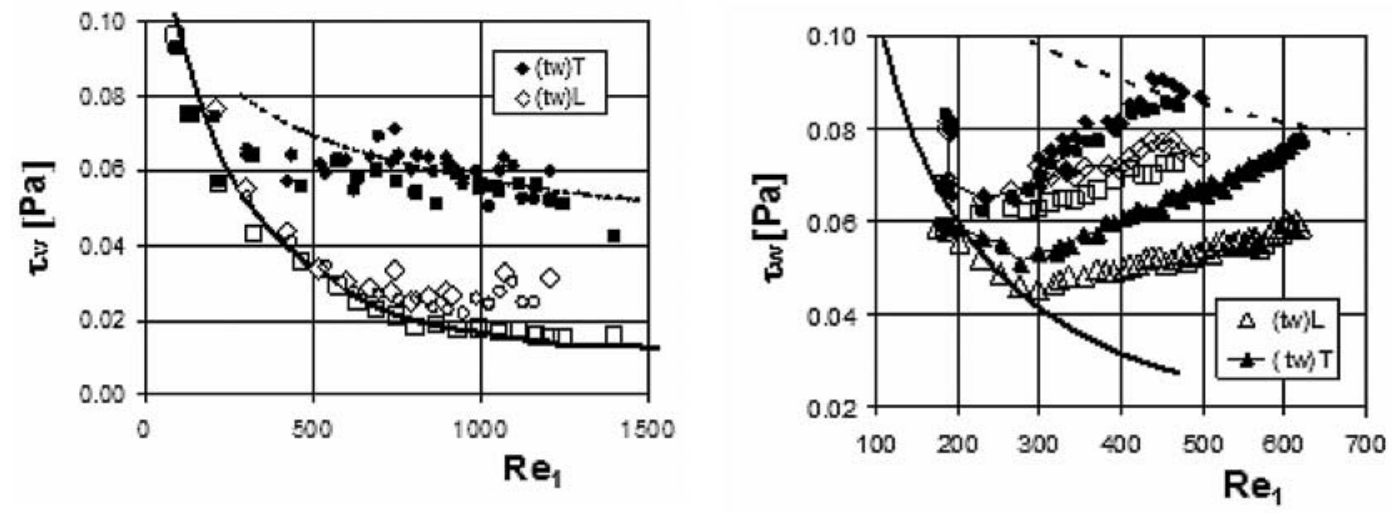

Fig. 7 Conditional mean wall frictions in smooth wall layer (left) and in rough wall layer (right) (Blasius solution - full line; Ludwieg and Tillmann distribution - dotted line)

pass transition is similar in both SWL and RWL. The smaller FST length parameter the faster turbulence decay occurs. Owing to this, intensity Iu in flow with larger $L e$ exceeds the local intensity in flow with smaller $L e$ in a given distance $x$, despite intensities are the same at $x=0$. Methods and procedures of measurement, processing and analysis developed for investigations of smooth wall layers are usable also in rough wall layers however transformation of the initial CTA signal record into the record of the instantaneous wall friction is much more laborious.

\section{Acknowledgement}

The authors gratefully acknowledge financial support of the Grant Agency of the Czech Republic, project GAP101/12/1271 and as well as the project AVOZ20760514.

\section{References}

[1] JONAS, P.: On the Role of the Length Scale in the by-pass Transition. ZAMM - Z. angew. Math. Mech. 77 (1997) S1, S145-S146.

[2] HLADIK, O., JONAS, P., MAZUR, O., URUBA, V.: Boundary Layer by-pass Transition by Joint Action of Surface Roughness and External Turbulence. Proc. Experimental Fluid Mechanics Conf. (Eds. Vit, T. and Dancova, P.), TU of Liberec (2010), 192-208.

[3] SCHLICHTING, H., GERSTEN, K.: Boundary-Layer Theory. Springer, Berlin, 2000.

[4] LUDWIEG, H., TILLMANN, W.: Untersuchungen uber die Wandschubspannung in turbulenten Reibungsschichten. Ing.-Archiv, Gd. 17 (1949), 288-299, borrowed from [3].

[5] JONAS, P., MAZUR, O., Uruba, V.: On the Receptivity of the by-pass Transition to the Length Scale of the Outer Stream Turbulence. Eur. J. Mech. B 19 (2000), 707-722. 


\section{COMMNICOIIONS}

[6] JONA, P., MAZUR, O. and URUBA, V.: Statistical Characteristics of the Wall Friction in a Flat Plate Boundary Layer through by-pass Transition. ZAMM Z. Angew. Math. Mech. 79 (1999), S691-S692.

[7] ZHANG, D. H., CHEW, Y. T., WINOTO, S. H.: Investigation of Intermittency Measurement Methods for Transitional Boundary. Exp. Thermal and Fluid Sci., 12 (1996), 433-443.

[8] HENDLEY, T. B., KEFFER, J. F.: Turbulent/non-turbulent Decision in an Intermittent Flow. J. Fluid Mech. 64 (1974), 625-644.

[9] ELSNER, W., WYSOCKI, M., DROBNIAK, S.: Determination of Production Rate of Turbulent Spots Using Wavelet Analysis. Chemical and Process Engineering, 27 (2006), 935-950.

[10] HLADIK, O., URUBA, V.: Analysis of Intermittent Signal, Mechanical Engineering J., June 2009, ISSN 1335-2938, 69-70.

[11] JONAS, P., ELSNER, W., MAZUR, O., URUBA, V., WYSOCKI, M.: Turbulent Spots Detection During Boundary Layer by-pass Transition. ERCOFTAC Bulletin 80 (2009), 16-19.

[12] NARASIMHA, R., The Laminar-turbulent Transition Zone in the Boundary Layer. Prog. Aerospace Sci. 22 (1985), 29-80.

[13] EMMONS, H.: The Laminar-turbulent Transition in a Voundary Layer - Part 1, J. Aeronaut. Sci. 18, (1951), 490-498.

[14] JOHNSON, M. W., FASHIFAR, A.: Statistical Properties of Turbulent Bursts in Transitional Boundary Layers. Int. J. Heat Fluid Flow 15 (1994), 283-290.

[15] FRANSSON, J. H. M., MATSUBARA, M., ALFREDSSON, P.H., Transition Induced by Free-stream Turbulence. J. Fluid Mech. 527 (2005), 1-25. 